\title{
Analysis of Infrared Wireless Links Employing Multibeam Transmitters and Imaging Diversity Receivers
}

\author{
Pouyan Djahani and Joseph M. Kahn, Fellow, IEEE
}

\begin{abstract}
We analyze the improvements obtained in wireless infrared (IR) communication links when one replaces traditional single-element receivers by imaging receivers and diffuse transmitters by multibeam (quasi-diffuse) transmitters. This paper addresses both line-of-sight (LOS) and nonline-of-sight (non-LOS) IR links. We quantify link performance in terms of the transmitter power required to achieve a bit error rate (BER) not exceeding $10^{-9}$ with $95 \%$ probability. Our results indicate that in LOS links, imaging receivers can reduce the required transmitter power by up to $13 \mathrm{~dB}$ compared to single-element receivers. In non-LOS links, imaging receivers and multibeam transmitters can reduce the required transmitter power by more than $20 \mathrm{~dB}$. Furthermore, we discuss the use of multibeam transmitters and imaging receivers to implement space-division multiple access (SDMA). In a representative example with two users transmitting at a power sufficient to achieve a BER not exceeding $10^{-9}$ with $95 \%$ probability in the absence of cochannel interference, when SDMA is employed, the system can achieve a BER not exceeding $10^{-9}$ with a probability of about $88 \%$.
\end{abstract}

Index Terms-Diversity methods, image sensors, optical communication, optimal receivers, optimal transmitters.

\section{INTRODUCTION}

$\mathbf{I}$ NFRARED (IR) radiation is a promising transmission medium for short-range indoor wireless communication systems [1]-[3]. In such systems, IR radiation offers several potential advantages over radio. IR emitters and detectors capable of high-speed operation are available at low cost. The IR spectral region offers a virtually unlimited bandwidth that is unregulated worldwide. IR light is blocked by walls or other opaque barriers, so that an IR transmission is confined to the room in which it originates. This signal confinement makes it easy to secure transmissions against casual eavesdropping, and it prevents interference between links operating in different rooms. Thus, IR wireless LANs can potentially achieve a very high aggregate capacity, and their design may be simplified,

Paper approved by O. K. Tonguz, the Editor for Optical Transmission Systems of the IEEE Communications Society. Manuscript received July 15, 1998 ; revised July 15, 1999. This work was supported by the National Science Foundation under Grant ECS-9632829, LG Electronics, Hewlett-Packard, and the University of California MICRO Program. This paper was presented in part at the IEEE International Conference on Global Communications (GLOBECOM), Rio de Janeiro, Brazil, December 1999.

P. Djahani was with the Department of Electrical Engineering and Computer Sciences,University of California, Berkeley, CA 94720-1770 USA. He is now with Cisco Systems, Inc., San Jose, CA 95134-1706 USA.

J. M. Kahn is with the Department of Electrical Engineering and Computer Sciences, University of California, Berkeley, CA 94720-1770 USA (e-mail: jmk@eecs.berkeley.edu).

Publisher Item Identifier S 0090-6778(00)10906-7. since transmissions in different rooms need not be coordinated. When an IR link employs intensity modulation with direct detection (IM/DD), the short carrier wavelength and large-area, square-law detector lead to efficient spatial diversity that prevents multipath fading [4]. By contrast, radio links are typically subject to large fluctuations in received signal magnitude and phase. Freedom from multipath fading greatly simplifies the design of IR links.

The IR medium has several potential drawbacks, however. Because IR cannot penetrate walls, communication from one room to another requires the installation of IR access points that are interconnected via a wired backbone. In many indoor environments there exists intense ambient IR noise, arising from sunlight, incandescent lighting and fluorescent lighting, which induces noise in an IR receiver. In virtually all short-range, indoor applications, IM/DD is the only practical transmission technique. The signal-to-noise ratio (SNR) of a direct-detection receiver is proportional to the square of the received optical power, implying that IM/DD links can tolerate only a comparatively limited path loss. Often, IR links must employ relatively high transmit power levels and operate over a relatively limited range. While the transmitter power level can usually be increased without fear of interfering with other users, transmitter power may be limited by concerns of power consumption and eye safety, particularly in portable transmitters.

Simple IR links may be classified based on the directionality of the receiver and transmitter, and on whether an uninterrupted line-of-sight (LOS) is necessary between the receiver and transmitter [2]. Currently, directed, LOS links, such as those standardized by the Infrared Data Association [5], are the most widely used IR links. Non-directed, nonline-of-sight (non-LOS) links, also known as diffuse links, are also becoming increasingly popular. Almost all current IR communication systems, whether LOS, non-LOS, directed, or nondirected, employ a single-element receiver. A single-element receiver [3] consists of an optical concentrator (usually nonimaging) whose output is coupled to a single photodetector. In a single-element receiver, the desired signal, ambient light noise, cochannel interference, and (often undesired) delayed multipath signal are combined into a single electrical signal.

Significant performance improvements can be achieved by using an angle-diversity receiver, which utilizes multiple receiving elements that are pointed in different directions [3], [6]. The photocurrents received in the various elements are amplified separately, and the resulting electrical signals 
can be processed in one of several ways, as described below. Angle-diversity receivers offer several advantages. They can achieve high optical gain over a wide field-of-view (FOV). They can significantly reduce the effects of ambient light noise, cochannel interference and multipath distortion, due to the fact that these unwanted signals are in many cases received from a different direction than the desired signal. An angle-diversity receiver can be implemented using multiple nonimaging elements that are oriented in different directions, as in [6]. Performance gains achieved by nonimaging angle-diversity receivers have been discussed in [6]-[9].

Implementation of angle diversity using nonimaging elements requires a separate optical concentrator for each receiving element, which may be excessively bulk and costly. Yun and Kavehrad proposed the fly-eye receiver [10], which consists of a single imaging optical concentrator (e.g., a lens) that forms an image of the received light on a collection of photodetectors, thereby separating signals that arrive from different directions. We will refer to this new design as an imaging angle-diversity receiver, or simply an imaging receiver [11]. The imaging design has two advantages over a nonimaging angle-diversity receiver. First, all photodetectors share a common concentrator, reducing size and cost. Second, all the photodetectors can be laid out in a single planar array, facilitating the use of a large number of receiving elements or pixels.

In nondirected, non-LOS links, the conventional diffuse transmitter utilizes a single broad beam aimed at reflecting surfaces within a room, such as the ceiling. Yun and Kavehrad proposed to replace this by the spot-diffusing transmitter [10], which employs multiple narrow beams pointed in different directions toward these reflecting surfaces. We will refer to the spot-diffusing transmitter as a multibeam or quasi-diffuse transmitter [11]. While the diffuse transmitter provides considerable immunity against beam blockage near the receiver, it yields a high path loss [3]. The quasi-diffuse transmitter is expected to reduce path loss compared to the diffuse transmitter, because the narrow beams experience little path loss travelling from the transmitter to the illuminated reflective surfaces [10].

Tang $e t$ al. presented an analysis comparing the performance of LOS links using imaging receivers to their counterparts employing single-element receivers [11]. They also compared non-LOS links using quasi-diffuse transmitters and imaging receivers to conventional diffuse/single-element links. They showed that considerable SNR gains can be achieved by using imaging receivers and quasi-diffuse transmitters. In [11], a number of simplifying assumptions were made in order to obtain closed-form analytical expressions for the link SNR. In this paper, we extend the analysis of [11] by relaxing these simplifying assumptions and using detailed numerical analysis to evaluate link performance. Effects considered for the first time here include the following: (a) nonideal imaging properties of a realistic lens; (b) overlap of the image spot with multiple detector pixels and relevant signal selection or combining techniques; (c) the precise number of beams required by the quasi-diffuse transmitter to achieve a specified range; (d) beam blockage in the quasi-diffuse transmitter; and (e) multipath distortion in links employing the quasi-diffuse transmitter. Our results confirm that imaging receivers and quasi-diffuse

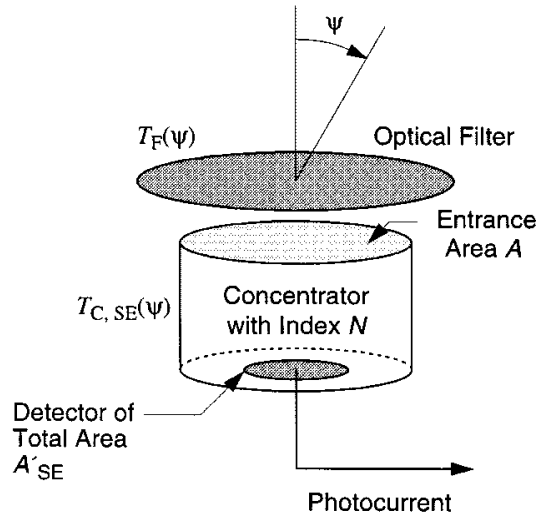

(a)

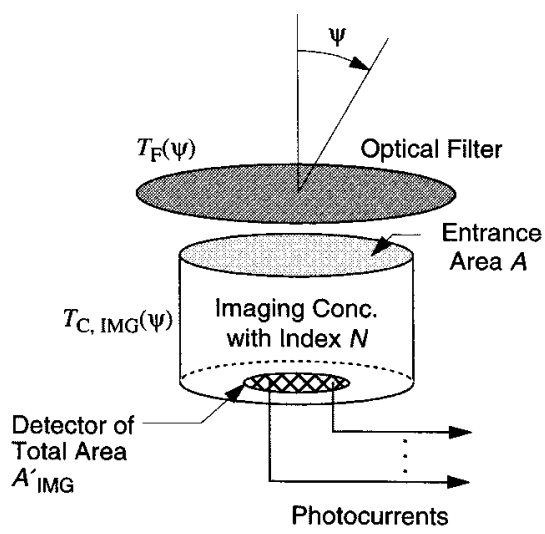

(b)

Fig. 1. Single-element and imaging optical receivers. (a) The single-element receiver employs a concentrator (usually nonimaging) in conjunction with a single detector. (b) The imaging receiver employs an imaging concentrator and a detector segmented into multiple pixels.

transmitters offer significant gains in link SNR and power efficiency. We also present a preliminary study showing that quasi-diffuse transmitters and imaging receivers may enable the use of space-division multiple access (SDMA) [3], wherein multiple transmitters located in close proximity can transmit simultaneously at the same wavelength with acceptably small mutual interference. We note that our group at the University of California, Berkeley, is experimenting currently with an imaging receiver utilizing a custom three-element lens and a 37-pixel photodetector array [12], [13]. Experimental work on imaging receivers is also in progress elsewhere [14], [15].

The remainder of this paper organized as follows. In Section II, we describe single-element and imaging receivers, and how they are modeled. In Section III, we present an analysis of the SNR and transmitter power requirements of LOS and non-LOS links. We discuss SDMA in Section IV, and provide concluding remarks in Section V.

\section{SingLe-Element AND IMAGing RECEIVERS}

Simplified schematics of single-element and imaging receivers are shown in Fig. 1. Both types of receivers typically employ an optical filter to attenuate unwanted ambient light. A single-element receiver, as shown in Fig. 1(a), employs an optical concentrator whose output is coupled to a single 
photodetector and single preamplifier. The imaging receiver, depicted in Fig. 1(b), utilizes an imaging concentrator that forms an image onto a photodetector segmented into multiple pixels, each equipped with a separate preamplifier. An image spot may overlap with one or more pixels. In this section, we describe how we model optical filters and concentrators, how we model imaging of the signal spot onto the pixels of the imaging receiver, and how we treat preamplifer thermal noise.

\section{A. Optical Filter and Concentrator}

Referring to Fig. 1, the total average ${ }^{1}$ optical power detected by an IR receiver (either single-element or imaging) at incidence angle $\psi$ (measured with respect to the receiver surface normal) is given by

$$
P=I(\psi) T_{F}(\psi) T_{C}(\psi) A \cos \psi
$$

where $I(\psi)$ is the incident irradiance $\left(\mathrm{W} / \mathrm{m}^{2}\right)$ at angle $\psi, T_{F}(\psi)$ is the filter transmission factor (W/W) at angle $\psi, T_{C}(\psi)$ is the optical concentrator transmission factor (W/W) at angle $\psi$, and $A$ is the receiver entrance area $\left(\mathrm{m}^{2}\right)$ at normal incidence. The transmission factors $T_{F}(\psi)$ and $T_{C}(\psi)$ always lie between 0 and 1. The optical concentrator has an acceptance semi-angle $\Psi_{a}$ such that when $\psi$ exceeds $\Psi_{a}$, the concentrator transmission factor rapidly approaches zero.

In our numerical analysis, we assume that both single-element and imaging receivers utilize an optical bandpass filter having a passband of width $\Delta \lambda=83 \mathrm{~nm}$, centered at $\lambda_{c}=833$ $\mathrm{nm}$. Measurements on an experimental prototype filter [12] indicate that for incidence angles $\psi$ between $0^{\circ}$ and $45^{\circ}$, the filter transmission factor is well modeled by $T_{F}(\psi)=0.8$.

The hemispherical lens [16] is a common nonimaging concentrator and has an acceptance semiangle $\Psi_{a}=90^{\circ}$. The compound-parabolic concentrator (CPC) [17] is another common nonimaging concentrator and has $\Psi_{a}<90^{\circ}$. In our analysis, the single-element receivers are assumed to employ CPCs having acceptance semiangle $\Psi_{a}=45^{\circ}$, refractive index $N=1.7$, and entrance area $A=9 \pi / 4 \mathrm{~cm}^{2}$. The transmission factor of the CPC is well modeled by $T_{C, \mathrm{SE}}=T\left[1+\left(\psi / \Psi_{a}\right)^{2 R}\right]^{-1}$, where $T=0.9$ and $R=13$ [17]. The CPC has an exit area $A_{\mathrm{SE}}^{\prime}=A \sin ^{2} \Psi_{a} / N^{2}$, which equals $1.22 \mathrm{~cm}^{2}$.

In our analysis of imaging receivers, the concentrator is modeled as a custom-built lens, which is described in detail in [12]. This lens utilizes three elements having index $N=1.7$; the rear element is index-matched directly to the antireflection-coated photodetector. The lens has an $f /$ number of 0.54 , and achieves an acceptance semiangle $\Psi_{a}=45^{\circ}$. The entrance aperture has a $3-\mathrm{cm}$ diameter, so that $A=9 \pi / 4 \mathrm{~cm}^{2}$. The image diameter is $d_{\mathrm{IMG}}=2.28 \mathrm{~cm}$, and the image spot diameter ranges from 1.4 to $6.6 \mathrm{~mm}$ for $\psi=0^{\circ}$ and $\psi=45^{\circ}$, respectively. Ray-tracing indicates that for $0 \leq \psi \leq \pi / 4$, the transmission factor of this imaging concentrator is well modeled by $T_{C, \mathrm{IMG}}(\psi)=-0.1982 \psi^{2}+0.0425 \psi+0.8778$, where $\psi$ is measured in radians.

We emphasize that in all calculations, both the single-element and imaging receivers employ equal entrance areas $A=9 \pi / 4$ $\mathrm{cm}^{2}$ and equal acceptance semiangles $\Psi_{a}=45^{\circ}$.

${ }^{1}$ Throughout this paper, the word "average" denotes an average over time.

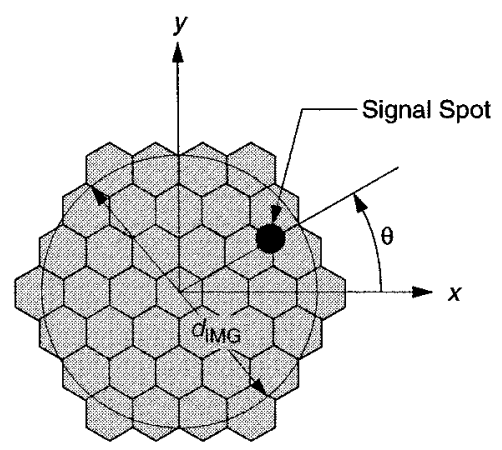

Fig. 2. The imaging receiver employs a photodetector segmented into multiple hexagonal pixels ( 37 pixels are shown here). The hexagonal pixel shape ensures that most signal image spots overlap with no more than three pixels, thereby helping maximize the SNR. Here, $d_{\text {IMG }}$ is the image diameter, and $\theta$ is the azimuthal angle from which the signal is incident.

\section{B. Photodetector Array and Image Spot}

In our analysis, the single-element receiver employs a single circular photodetector having area $A_{\mathrm{SE}}^{\prime}=1.22 \mathrm{~cm}^{2}$. The imaging receiver employs a detector array segmented into $J$ equal-sized hexagonal pixels, as shown in Fig. 2. We assume that there are no gaps between the pixels. Using hexagonal pixels ensures that, under most circumstances, the signal image spot falls on no more than three pixels, thereby maximizing the receiver SNR (see Section III). The possible number of pixels $J$ in an arrangement similar to Fig. 2 is given by $J=2 \sum_{i=1}^{n}(n-i-1)-2 n+1=3\left(n^{2}-n\right)+1$, where $n$ is any integer greater than or equal to 2 . For the imaging receivers analyzed in this paper, the value of $n$ ranges from 2 to 20 , corresponding to $J$ ranging from 7 to 1141 pixels. As indicated in Fig. 2, the detector array size is chosen to be just large enough to cover the entire image of diameter $d_{\mathrm{IMG}}$. Accordingly, the area of an individual pixel is given by

$$
A_{\mathrm{IMG}, i}^{\prime}=\frac{3 \sqrt{3}}{8} \frac{d_{\mathrm{IMG}}^{2}}{\left(\frac{1+\sqrt{1+4 \frac{J-1}{3}}}{2}+\left\lfloor\frac{-1+\sqrt{1+4 \frac{J-1}{3}}}{4}\right\rfloor\right)^{2}} .
$$

Note that as $J$ becomes large, this becomes much smaller than $A_{\mathrm{SE}}^{\prime}$.

In the imaging receiver, when the signal image spot overlaps more than one pixel, the $i$ th pixel receives a fraction $f_{i}(\psi, \theta)$ of the total power. The overlap factor $f_{i}(\psi, \theta)$ depends on the polar and azimuthal incidence angles $\psi$ and $\theta$, which are specified in Figs. 1 and 2, respectively. In our calculations, the values of $f_{i}(\psi, \theta)$ are determined by numerical ray tracing in the threeelement lens [12]. The average optical signal power received in the $i$ th pixel is thus given by

$$
P_{\mathrm{IMG}, i}=f_{i}(\psi, \theta) P_{\mathrm{IMG}}
$$

where $P_{\mathrm{IMG}}$ is the average optical signal power received by the imaging receiver, given by (1).

\section{Preamplifier Noise}

We follow the analysis of Smith and Personick in computing the receiver noise [18], [19]. This analysis assumes on-off keying $(\mathrm{OOK})$ with rectangular transmitted pulses of duration equal to the bit period, and a receiver filter that equalizes the received pulse to have a raised-cosine spectrum with $100 \%$ 
excess bandwidth. We assume the use of p-i-n photodetectors in conjunction with FET-based transimpedance preamplifiers. For simplicity, we neglect FET gate leakage and $1 / f$ noise. We first consider an imaging receiver employing separate preamplifiers for each pixel. The noise variance referred to the input of the $i$ th pixel is given approximately by

$$
\begin{aligned}
\sigma_{\text {tot }, i}^{2} \approx 2 q r P_{b, i} I_{2} B+\frac{4 k T}{R_{F}} & I_{2} B \\
& +\frac{16 \pi^{2} k T \Gamma}{g_{m}}\left(C_{d}+C_{g}\right)^{2} I_{3} B^{3} .
\end{aligned}
$$

In the first term, which represents ambient-induced shot noise, $q$ is the electronic charge, $r$ is the detector responsivity, $P_{b, i}$ is the ambient light power detected by the $i$ th pixel, $I_{2}=0.562$, and $B$ is the bit rate. The second term represents thermal noise from the feedback resistor; $k$ is Boltzmann's constant, $T$ is absolute temperature, and $R_{F}$ is the feedback resistance. In the third term, which describes thermal noise from the FET channel resistance, $\Gamma$ is the FET channel noise factor, $g_{m}$ is the FET transconductance, $C_{d}$ is the capacitance of a single detector pixel, $C_{g}$ is the FET gate capacitance, and $I_{3}=0.0868$.

In order to determine explicitly how the three noise terms in (4) depend on the pixel size, we assume that the photodetector has a fixed capacitance per unit area $\eta$, i.e., $C_{d}=\eta A^{\prime}$, where $A^{\prime}$ is the detector area. For simplicity, we assume that $c_{g} \ll$ $C_{d}$. We assume that the transimpedance amplifier has a limited open-loop voltage gain $G$. In order to minimize the noise, it is desirable to maximize $R_{F}$, but if the preamplifier is to achieve a 3-dB cutoff frequency equal to $B$, then we must impose the condition $R_{F}=G / 2 \pi B C_{d}$. Then (4) becomes

$$
\begin{aligned}
\sigma_{\mathrm{tot}, i}^{2} \approx 2 q r P_{b, i} I_{2} B+\frac{8 \pi k T}{G} \eta A^{\prime} I_{2} B^{2} & \\
& +\frac{16 \pi^{2} k T T}{g_{m}} \eta^{2} A^{2} I_{3} B^{3} .
\end{aligned}
$$

In order to model noise in a single-element receiver, we employ (4) or (5), interpreting all quantities to pertain to the single detector and preamplifier. Use of an imaging receiver with many small pixels will typically reduce all three terms in (5), as compared to a single-element receiver. The first term is reduced because the small FOV associated with a small pixel size diminishes the received ambient light power, while the second and third terms are reduced because a small pixel size reduces the preamplifier input capacitance. In our numerical examples, we choose the following parameter values: $T=295 \mathrm{~K}, r=0.54$ $\mathrm{A} / \mathrm{W}, G=10, g_{m}=30 \mathrm{mS}, \Gamma=1.5, \eta=112 \mathrm{pF} / \mathrm{cm}^{2}$, and $B=30 \mathrm{Mb} / \mathrm{s}^{2}$

\section{ANALYSIS OF IR LINKS}

In this section, we describe combining and selection techniques for imaging receivers and compute the resulting SNRs. We then analyze the performance of LOS and non-LOS links,

\footnotetext{
${ }^{2}$ In practice, choice of an excessively high value of $R_{F}$ may make the receiver dynamic range unacceptably small. Also, with excessively high values of $R_{F}$, achieving a receiver 3-dB cutoff frequency equal to the bit rate may require a value of $G$ so high that the receiver becomes unstable. For the numerical parameters considered here, dynamic range and stability should be acceptable. If, in practice, it is not possible to use a value of $G$ high enough that the 3-dB cutoff equals the bit rate, the receiver may employ an equalizer after the transimpedance preamplifier.
}

computing the transmit power required to achieve a BER not exceeding $10^{-9}$ with $95 \%$ probability. We also discuss the effects of multipath-induced intersymbol interference (ISI) on non-LOS links.

\section{A. Selection and Combining Techniques}

In a single-element receiver, following [18] and [19], the average electrical SNR is given by

$$
\mathrm{SNR}_{\mathrm{SE}}=\frac{\left(r P_{\mathrm{SE}}\right)^{2}}{\sigma_{\mathrm{tot}}^{2}} \text {. }
$$

In an imaging receiver, the received signal spot may be divided between several pixels, as shown in Fig. 2. We consider two ways to process the resulting electrical signals, namely, select-best (SB) and maximal-ratio combining (MRC). The SB method chooses the pixel in the detector array that has the highest SNR. The SNR using SB is given by

$$
\mathrm{SNR}_{\mathrm{IMG}, \mathrm{SB}}=\underset{i}{\max }\left(\frac{r^{2} P_{\mathrm{IMG}, i}^{2}}{\sigma_{\mathrm{tot}, i}^{2}}\right), \quad 1 \leq i \leq J
$$

Assuming a fixed area for the detector array, as the number of pixels in an imaging receiver increases, the area of each pixel decreases, and the noise variance per pixel $\sigma_{\text {tot }, i}^{2}$ decreases, tending to increase the SNR. As the pixel area becomes smaller than the signal spot area, the signal spot overlaps with multiple pixels and each pixel receives a smaller fraction of the total received signal power, tending to decrease the SNR.

In MRC, signals from the $J$ pixels are combined using weights equal to $w_{i}=r P_{\mathrm{IMG}, i} / \sigma_{\mathrm{tot}, i}^{2}, 1 \leq i \leq J$, thereby maximizing the SNR of the weighted sum. The SNR obtained using MRC is given by

$$
\mathrm{SNR}_{\mathrm{IMG}, \mathrm{MRC}}=\frac{\left(\sum_{i=1}^{J} r P_{\mathrm{IMG}, i} \frac{r P_{\mathrm{IMG}, i}}{\sigma_{\mathrm{tot}, i}^{2}}\right)^{2}}{\sum_{i=1}^{J}\left(\frac{r P_{\mathrm{IMG}, i}}{\sigma_{\mathrm{tot}, i}^{2}}\right)^{2} \sigma_{\mathrm{tot}, i}^{2}}=\sum_{i=1}^{J} \frac{r^{2} P_{\mathrm{IMG}, i}^{2}}{\sigma_{\mathrm{tot}, i}^{2}} .
$$

It is easy to see that the SNR achieved with MRC is always at least as high as under the SB method. For a fixed pixel size and noise variance per pixel, in order to maximize the worst SNR achieved as the signal spot is moved to various positions in the pixel array, it is necessary to minimize the maximum number of pixels the spot can illuminate. Use of hexagonal pixels insures that the spot illuminates no more than three pixels, provided that the spot is sufficiently small relative to the pixel size. With $\mathrm{MRC}$, as with SB, as the number of pixels is increased, the noise variance per pixel decreases, tending to increase the SNR. As with $\mathrm{SB}$, when the pixel area becomes smaller than the signal spot area, each pixel receives a smaller fraction of the total signal power, tending to decrease the SNR.

For both SB and MRC, assuming OOK with equiprobable zeros and ones, and assuming that ISI is negligible, the BER is given by

$$
\mathrm{BER}=Q(\sqrt{\mathrm{SNR}})
$$

where $Q(x)=(2 \pi)^{-1 / 2} \int_{x}^{\infty} \exp \left(-z^{2} / 2\right) d z$. For example, in order to achieve a BER of $10^{-9}$, an SNR of $36(15.6 \mathrm{~dB})$ is required. 


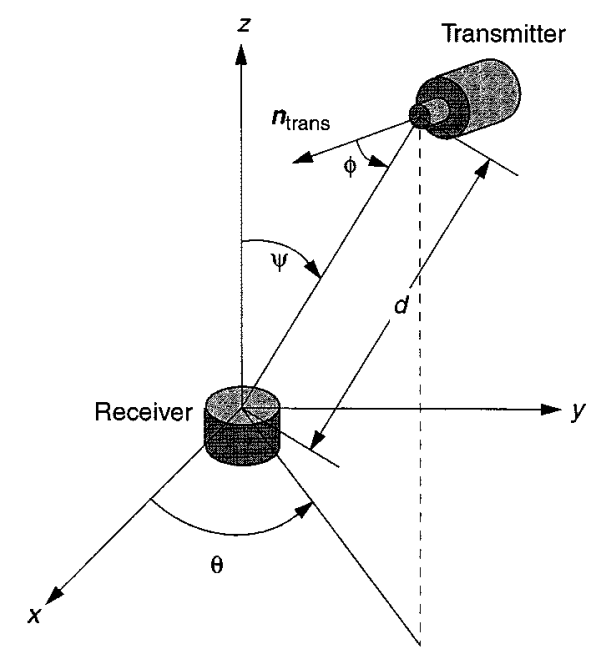

Fig. 3. Geometry used in LOS link calculations.

\section{B. LOS Link Analysis}

LOS links require an uninterrupted LOS between the receiver and the transmitter. A typical configuration for an LOS IR link is shown in Fig. 3. LOS links implement directional transmitters, which minimize multipath distortion due to reflections from walls and other surfaces, thereby minimizing ISI. We assume LOS links use OOK at a bit rate of $30 \mathrm{Mb} / \mathrm{s}$, and we neglect multipath ISI, so that the BER can be calculated using (9). As we will show later, at this bit rate, the effects of multipath ISI are negligible even in the non-LOS links analyzed.

We model the transmitter as a generalized Lambertian transmitter, with a radiant intensity $(\mathrm{W} / \mathrm{sr})$ given by

$$
R(\phi)=P_{\mathrm{trs}, \mathrm{LOS}} \frac{(n+1)}{2 \pi} \cos ^{n} \phi
$$

where $P_{\mathrm{trs}, \mathrm{LOS}}$ is the total average power of the transmitter, $\phi$ is the angle with respect to the transmitter surface normal, and $n$ is related to the half-power semiangle $\Phi_{1 / 2}$ of the transmitter by $n=-\ln 2 / \ln \left(\cos \left(\Phi_{1 / 2}\right)\right)$. Furthermore, we assume the transmitter has a half-power semiangle $\Phi_{1 / 2}$ equal to $45^{\circ}$, corresponding to an $n=2$.

We analyze LOS links employing either single-element receivers (LOS/SE) or imaging receivers (LOS/IMG). Both types of receivers acceptance semiangles $\Psi_{a}=45^{\circ}$. For both types of links, we assume the geometry shown in Fig. 3. We fix the position and orientation of the receiver. For a given transmitter-receiver separation $d$, we vary the position and orientation of the transmitter. We let $\phi$, the angle between the transmitter surface normal $\mathbf{n}_{\text {trans }}$ and the line connecting the receiver and the transmitter, is uniformly distributed between $0^{\circ}$ and $\Phi_{1 / 2}$. Also, we let $\psi$ (the angle between the receiver surface normal and the line connecting the receiver and the transmitter) be uniformly distributed between $0^{\circ}$ and $\Psi_{a}$. The single-element receiver has rotational symmetry, so that the azimuthal angle $\theta$ is irrelevant.
For the imaging receiver, we let $\theta$ be uniformly distributed between $0^{\circ}$ and $360^{\circ}$.

In an imaging receiver, the total signal power is distributed among the $J$ pixels. The average power detected by the $i$ th pixel is given by (11), shown at the bottom of the page. The total average signal power detected by a single-element receiver can be obtained using (11) by omitting $f_{i}(\psi, \theta)$ and replacing $T_{C, \mathrm{IMG}}(\psi)$ by $T_{C, \mathrm{SE}}(\psi)$.

We assume that in LOS links, the ambient light noise is dominated by skylight, which is described by a power spectral density $B_{\text {sky }}=43 \mathrm{~mW} /\left(\mathrm{m}^{2} \mathrm{sr} \cdot \mathrm{nm}\right)$ in the receiver optical filter passband

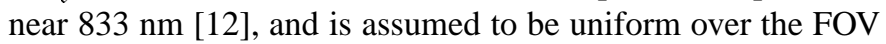
of the receiver. The total background noise in a single-element receiver due to the sky is given by

$$
P_{b, \mathrm{LOS}, \mathrm{SE}}=\pi A B_{\mathrm{sky}} \Delta \lambda \int_{0}^{\Psi_{a}} T_{F}(\psi) T_{C, \mathrm{SE}}(\psi) \sin (2 \psi) d \psi
$$

Likewise, for an imaging receiver, the total background noise in the $i$ th pixel due to the sky is approximately given by

$$
\begin{aligned}
P_{b, \mathrm{LOS}, \mathrm{IMG}, i} \approx 4 \pi A B_{\mathrm{sky}} \Delta \lambda T_{F}\left(\psi_{i}\right) T_{C, \mathrm{IMG}}\left(\psi_{i}\right) \\
\times \cos \psi_{i} \sin ^{2}\left(\frac{\Psi_{a, i}}{2}\right)
\end{aligned}
$$

where $\Psi_{a, i}$ is the acceptance angle of the $i$ th pixel, and $\psi_{i}$ is the angle of incidence of a ray with respect to the receiver normal, which strikes the detector array at the center of the $i$ th pixel.

At a fixed transmitter-receiver separation $d$, we consider a large ensemble of different values of $\phi, \psi$, and $\theta$ to estimate the complementary cumulative distribution function (ccdf) of the SNR. We then use the ccdf to determine the transmitter power required to achieve a BER not exceeding $10^{-9}$ with $95 \%$ probability. Fig. 4(a) shows the transmitter power requirement in LOS/SE and LOS/IMG links operating at $30 \mathrm{Mb} / \mathrm{s}$ for $d=4$ $\mathrm{m}$ and $d=8 \mathrm{~m}$, as a function of the number of pixels in the detector array. For both $d=4 \mathrm{~m}$ and $d=8 \mathrm{~m}$, using either SB or MRC, the simplest imaging receiver (seven pixels) requires $3.7 \mathrm{~dB}$ less transmit power than a single-element receiver. As the number of pixels in the imaging receiver is increased, the power requirement decreases further. At 1141 pixels, it reaches $13 \mathrm{~dB}$ for both values of $d$ and both combining techniques. Fig. 4(a) shows that the transmit power requirement with MRC is always less than or equal to that with SB. While the advantage of MRC over SB increases with the number of pixels, it is less than $1 \mathrm{~dB}$ in all cases.

Fig. 4(b) shows the transmitter power requirements in $30-\mathrm{Mb} / \mathrm{s}$ LOS links, as a function of the transmitter-receiver separation $d$, for a single-element receiver and for imaging receivers of 37 and 1141 pixels. For $d$ ranging from 1 to $8 \mathrm{~m}$, the 37-pixel imaging receiver using MRC requires at least $6.8 \mathrm{~dB}$ less power than the single-element receiver. With SB,

$$
P_{\mathrm{LOS}, \mathrm{IMG}, i}= \begin{cases}P_{\mathrm{trs}, \mathrm{LO}} \frac{3 A}{2 \pi d^{2}} \cos ^{2} \phi T_{F}(\psi) f_{i}(\psi, \theta) T_{C, \mathrm{IMG}}(\psi) \cos \psi, & 0 \leq \psi \leq \Psi_{a} \\ 0, & \psi>\Psi_{a}\end{cases}
$$




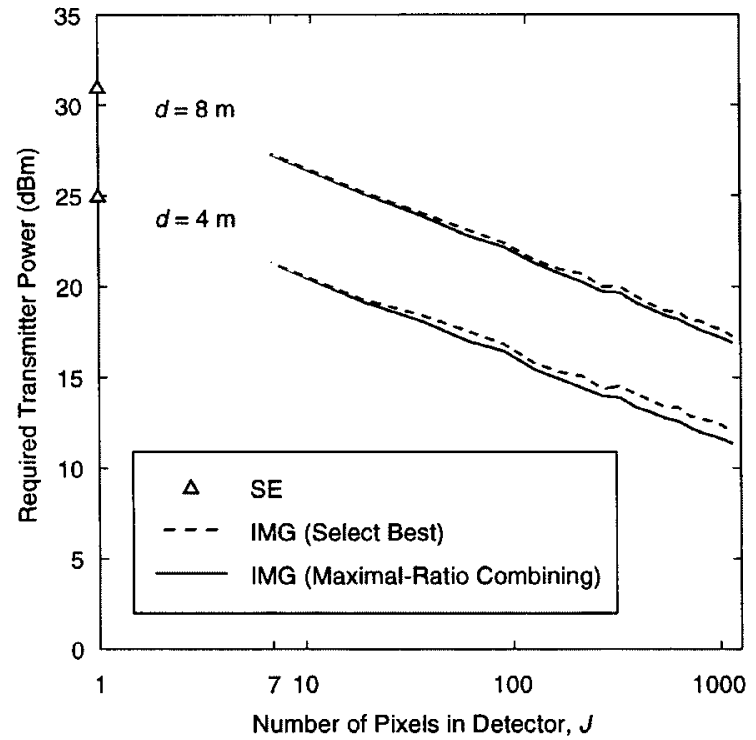

(a)

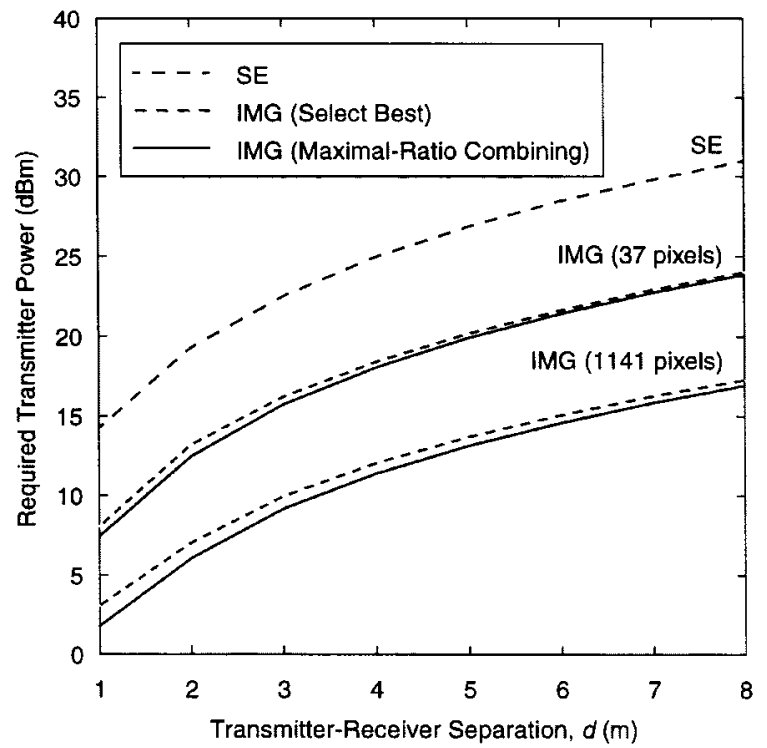

(b)

Fig. 4. Transmitter power required to achieve a BER not exceeding $10^{-9}$ with 95\% probability in LOS links using single-element (SE) and imaging (IMG) receivers: (a) as a function of the number of pixels in the detector array and (b) as a function of the distance between receiver and transmitter. All links use on-off keying and operate at $30 \mathrm{Mb} / \mathrm{s}$.

the reduction is at least $6.1 \mathrm{~dB}$. When the number of pixels is 1141 , the power requirements are decreased by 12.4 and $11.8 \mathrm{~dB}$ with MRC and SB, respectively.

\section{Non-LOS Link Analysis}

Unlike LOS links, non-LOS links do not require an uninterrupted LOS between the transmitter and receiver, but rely on reflections of IR radiation from surfaces. In this section, we consider two types of transmitters used in non-LOS links. The first type of transmitter is the diffuse transmitter, which employs a single wide beam to illuminate surfaces, as shown in Fig. 5(a). Diffuse transmitters provide excellent immunity

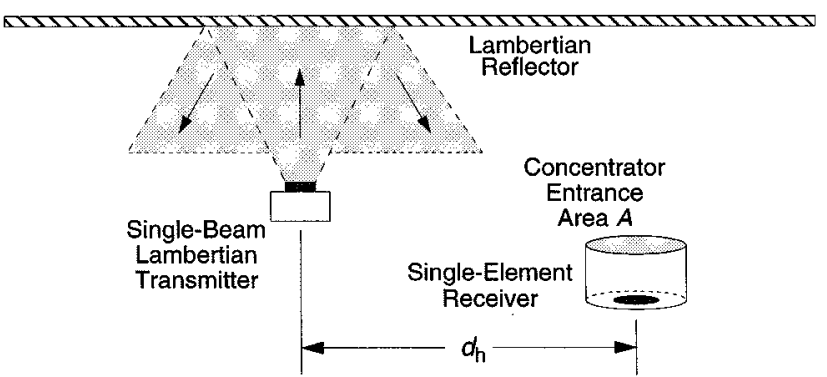

(a)

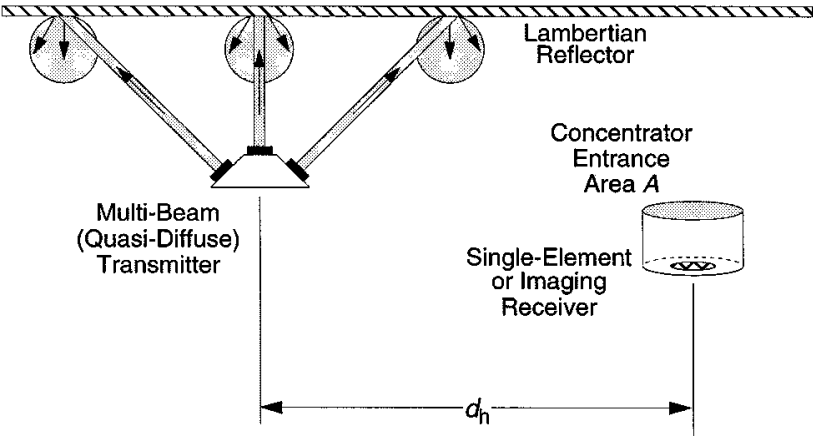

(b)

Fig. 5. Non-LOS infrared links. (a) A diffuse transmitter illuminates the ceiling (or other surfaces) with a single wide beam. (b) A quasi-diffuse transmitter illuminates the ceiling with multiple narrow beams, which form a regular lattice of spots on the ceiling.

against blockage, but suffer from high path loss and significant multipath distortion. The second type of transmitter, the quasi-diffuse transmitter, illuminates the ceiling with multiple narrow beams, ${ }^{3}$ as illustrated in Fig. 5(b). These beams form a regular lattice of spots on the ceiling, as shown in Fig. 6. ${ }^{4}$ Quasi-diffuse transmitters offer lower path loss than diffuse transmitters, because the narrow beams experience very little path loss travelling from the transmitter to the illuminated reflective surfaces [10]. We consider two types of quasi-diffuse transmitters, referred to as "Type I" and "Type II," and illustrated in Fig. 6(a) and (b), respectively. With a Type I transmitter, at least one signal spot always lies within the receiver FOV. The Type I transmitter does not provide immunity against blockage of the path between the transmitter and ceiling or between the ceiling and receiver. The Type II transmitter is designed so that at least two signal spots always lie within the receiver FOV, making it possible for the link to operate if a single spot is obstructed.

Both single-element and imaging receivers can be used in conjunction with diffuse and quasi-diffuse transmitters in non-LOS links. In our analysis, we will consider the following: (a) diffuse transmitters with single-element receivers (DIF/SE); (b) quasi-diffuse transmitters with single-element receivers

\footnotetext{
${ }^{3}$ It should be noted that, although the quasi-diffuse transmitter uses narrow beams, eye safety can always be insured by making the beam diameter sufficiently large. Furthermore, the power required in each beam is relatively low (of the order of milliwatts) and modest beam divergence is acceptable [12].

${ }^{4}$ The spot lattices shown in Fig. 6 assume that the transmitter is far from any walls. When the transmitter is placed sufficiently close to a wall, some of the beams will illuminate the wall, instead of the ceiling.
} 

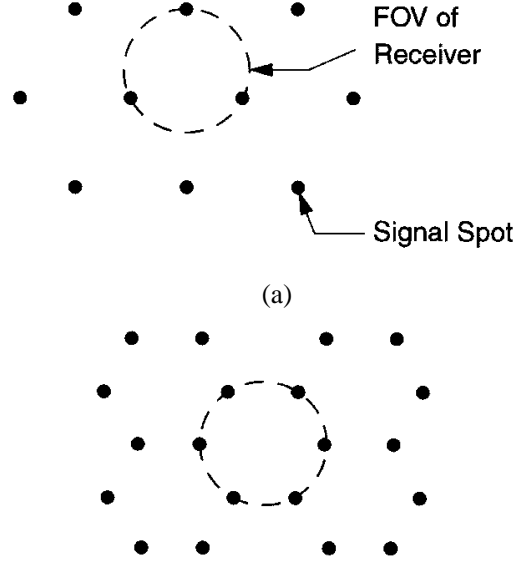

(b)

Fig. 6. Lattice of spots formed on the ceiling by quasi-diffuse transmitters. The configurations shown minimize the number of spot required per unit area. (a) Type I quasi-diffuse transmitter, which is not immune to shadowing (blockage of the signal spot). At least one signal spot is always in the FOV of the receiver (b) Type II quasi-diffuse transmitter. Because at least two signal spots always lie within the receiver FOV, the Type II transmitter can tolerate blockage of one signal spot.

(QDIF/SE); and (c) quasi-diffuse transmitters with imaging receivers (QDIF/IMG). ${ }^{5}$

1) Link Configurations: The room shown in Fig. 7(a), representative of a typical medium-sized office [6], is used to analyze non-LOS links. The room has length, width, and height given by $X_{\mathrm{MAX}}=6 \mathrm{~m}, Y_{\mathrm{MAX}}=10 \mathrm{~m}$, and $Z_{\mathrm{MAX}}=4$ $\mathrm{m}$, respectively. The west wall of the room is a single large window. All room boundaries are modeled as Lambertian reflectors. All surfaces have reflectivities of 0.7 , except the floor, which has a reflectivity of 0.2 . The room boundaries act as Lambertian sources of background ambient light. According to measurements [6], in the receiver optical filter passband near 833 $\mathrm{nm}$ [12], the west and east walls have spectral radiant emittances $S_{W}=0.20 \mathrm{~W} /\left(\mathrm{m}^{2} \mathrm{~nm}\right)$ and $S_{E}=0.10 \mathrm{~W} /\left(\mathrm{m}^{2} \mathrm{~nm}\right)$, respectively. The spectral radiant emittance from the ceiling $\left(S_{C}\right)$ is assumed to vary linearly from $0.03 \mathrm{~W} /\left(\mathrm{m}^{2} \mathrm{~nm}\right)$ at the west edge to $0.01 \mathrm{~W} /\left(\mathrm{m}^{2} \mathrm{~nm}\right)$ at the east edge. The spectral radiant emittance from the north wall $\left(S_{N}\right)$, the south wall $\left(S_{S}\right)$, and the floor $\left(S_{F}\right)$ vary linearly from $0.02 \mathrm{~W} /\left(\mathrm{m}^{2} \mathrm{~nm}\right)$ at the west edge to $0.01 \mathrm{~W} /\left(\mathrm{m}^{2} \mathrm{~nm}\right)$ at the east edge. Eight tungsten flood lamps are placed on the ceiling of the room, at coordinates $(x, y, z)=(1.5,1.25,4),(1.5,3.75,4),(1.5,6.25,4)$, $(1.5,8.75,4),(4.5,1.25,4),(4.5,3.75,4),(4.5,6.25,4)$, and $(4.5,8.75,4)$ These lamps can be modeled as Lambertian transmitters of order $n=2.0$ with a total power spectral density $p_{\text {lamp }}=0.037 \mathrm{~W} / \mathrm{nm}$ within the receiver filter passband [6]. We denote the region occupied by the $j$ th flood lamp as $R_{\text {lamp }}, j$, which is taken to be a circular region of radius $r_{\text {lamp }}=5 \mathrm{~cm}$, over which the power $p_{\text {lamp }}$ is distributed uniformly.

\footnotetext{
${ }^{5}$ We do not consider combining diffuse transmitters with imaging receivers in this paper. Imaging receivers are significantly more complex to implement than single-element receivers, while quasi-diffuse transmitters are not much more complex than diffuse transmitters. With imaging receivers, quasi-diffuse transmitters provide much better performance than their diffuse counterparts, because the former transmitters concentrate the signal in a small spot, making possible enhanced rejection of undesired noise, multipath and cochannel interference.
}

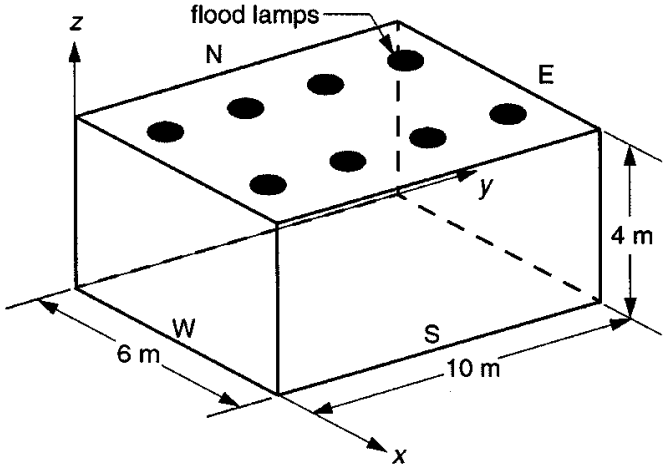

(a)

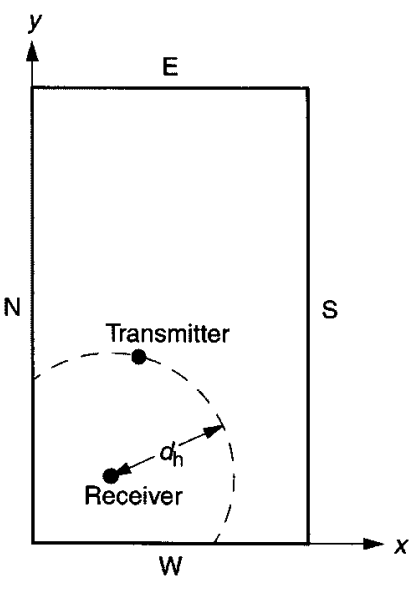

(b)

Fig. 7. Room model used for analyzing non-LOS links: (a) The west wall is a single, large window. Eight, 100-W incandescent flood lamps are mounted on the ceiling. (b) Position of transmitter and receiver within room.

Both single-element and imaging receivers have acceptance semiangles $\Psi_{a}=45^{\circ}$. As shown in Fig. 7(b), the receiver is placed at a fixed position at $\left(X_{R}, Y_{R}, Z_{R}\right)=(1.5,1.25,1)$, directly under one of the flood lamps, and faces upwards. ${ }^{6}$ Calculations indicate that this location is subject to the highest ambient light noise within the room (or very nearly so), thus representing a worst-case receiver location. As before, the imaging receiver has an azimuthal orientation as in Fig. 2, while the azimuthal orientation of the single-element receiver is irrelevant.

The transmitter (diffuse or quasi-diffuse) is kept at a fixed height of $1 \mathrm{~m}$ above the floor. For a given horizontal transmitter-receiver separation $d_{h}$, the $x$ and $y$ coordinates of the transmitter are modeled as random variables, which are uniformly distributed on that portion of the circumference of a circle of radius $d_{h}$, lying parallel to the $x$ - $y$ plane and centered at $\left(X_{R}, Y_{R}, Z_{R}\right)$, that lies within the room, as shown in Fig. 7(b). We model the azimuthal orientation of the quasi-diffuse transmitter (around its surface normal) as a random variable, uni-

\footnotetext{
${ }^{6}$ It should be noted that, because the receiver faces upwards and has an acceptance semi-angle $\Psi_{a}=45^{\circ}$, no region of the floor will be in its FOV. Consequently, the floor does not directly reflect any signal or ambient light to the receiver, and can be neglected in our calculations. However, the floor is taken into account when calculating the impulse response $h(t)$ that describes multipath propagation in the room.
} 
formly distributed between 0 and $2 \pi$, while the azimuthal orientation of the diffuse transmitter is irrelevant.

2) SNR Analysis of Non-LOS Links: For the link configuration shown in Fig. 7(b), we first calculate the ambient light noise in SE and IMG receivers, then determine the signal power received in DIF/SE, QDIF/IMG, and QDIF/SE links. Using these noise and signal components, we evaluate the SNR and transmit power requirement for each of the three link designs.

Let $R_{\text {Surface,IMG, } i}$ denote the region of the room boundary within the FOV of the $i$ th pixel of the imaging receiver. The ambient light power from room boundaries detected by the $i$ th pixel is given by

$$
\begin{aligned}
& P_{b, \text { Surface,IMG }, i} \\
& =\int_{\substack{(x, y, z) \in \\
R_{\text {Surface,IMG }, i}}} \frac{S_{\text {Surface }}(x, y, z) \Delta \lambda A T_{F}(\phi) f_{i}(\psi, \theta) T_{C, \mathrm{IMG}}(\psi)}{\pi\left[\left(x-X_{R}\right)^{2}+\left(y-Y_{R}\right)^{2}+\left(z-Z_{R}\right)^{2}\right]} \\
& \quad \cdot \cos (\phi) \cos (\psi) d s
\end{aligned}
$$

where $S_{\text {Surface }}(x, y, z)$ is the spectral radiant emittance of the surface and $d s$ is a differential surface element. The angle $\psi$ is given by

$$
\psi=\cos ^{-1}\left(\frac{z-Z_{R}}{\sqrt{\left(x-X_{R}\right)^{2}+\left(y-Y_{R}\right)^{2}+\left(z-Z_{R}\right)^{2}}}\right)
$$

for all surfaces. For the ceiling, $\phi$ equals $\psi$; for the walls, $\phi=$ $90^{\circ}-\psi$.

We let $R_{\text {Surface,SE }}$ denote the region of the room boundary lying within the FOV of the single-element receiver. The ambient light power from room boundaries detected by a single-element receiver is calculated using (14) by omitting $f_{i}(\psi, \theta)$ and replacing $R_{\text {Surface,IMG }}, i$ with $R_{\text {Surface,SE }}$.

The power from the $j$ th flood lamp detected by the $i$ th pixel of the imaging receiver is given by

$$
\begin{aligned}
& P_{b, \text { lamp }, j, \mathrm{IMG}, i} \\
& =\int \\
& (x, y, z) \in \\
& R_{\mathrm{lamp}, \mathrm{j}} \cap R_{C, \mathrm{IMG},} \\
& \cdot \frac{3 p_{\text {lamp }} \Delta \lambda A T_{F}(\psi) f_{i}(\psi, \theta) T_{C, \mathrm{IMG}}(\psi)}{2 \pi^{2} r_{\text {lamp }}\left[\left(x-X_{R}\right)^{2}+\left(y-Y_{R}\right)^{2}+\left(Z_{\mathrm{MAX}}-Z_{R}\right)^{2}\right]} \\
& \cdot \cos (\phi)^{2} \cos (\psi) d s
\end{aligned}
$$

where $R_{C, \mathrm{IMG}, i}$ is the region on the ceiling in the FOV of the $i$ th pixel, and $\psi$ and $\phi$ are equal, and are given by (15).

The background noise detected by a single-element receiver from the $j$ th flood lamp is calculated using (16) by omitting $f_{i}(\psi, \theta)$ and replacing $R_{C, \mathrm{IMG}, i}$ with $R_{C, \mathrm{SE}}$, the region on the ceiling in the FOV of the single-element receiver.

The total ambient light noise in a receiver is the sum of contributions from the room boundary surfaces and the flood lamps. In the imaging receiver, the total ambient light power in the $i$ th pixel is given by

$$
P_{b, \mathrm{IMG}, i}=P_{b, \text { Surface,IMG }, i}+\sum_{j=1}^{8} P_{b, \text { lamp }, j, \mathrm{IMG}, i} .
$$

The total ambient light power in the single-element receiver is obtained using an expression analogous to (17).

We consider the diffuse transmitter in conjunction with a single-element receiver. The diffuse transmitter is modeled as having a generalized Lambertian radiant intensity given by (10) with order $n=2$. It is positioned as illustrated in Fig. 7(b) and pointed upward at the ceiling. The illuminated regions of the ceiling and walls are assumed to act as Lambertian reflectors. The average signal power reflected by the ceiling and detected by the single-element receiver is given by (18), shown at the bottom of the page, where $\left(X_{T}, Y_{T}, Z_{T}\right)$ is the position of the transmitter, $\sigma_{C}$ is the reflectivity of the ceiling, $P_{\mathrm{trs}, \mathrm{DIF}}$ is the average power emitted by the diffuse transmitter, and $\psi$ is given by (15). Similarly, the average signal power reflected by a wall and detected by the single-element receiver is given by (19), shown at the bottom of the page, where $R_{\mathrm{Wall}, \mathrm{SE}}$ is the region of the wall lying in the FOV of the receiver, and $\sigma_{\text {Wall }}$ is the reflectivity of the wall.

We consider quasi-diffuse transmitters with both imaging and single-element receivers. As previously mentioned, the quasidiffuse transmitter emits multiple narrow beams, which illuminate a regular lattice of spots on the ceiling, assuming that the transmitter is far from any walls (see Fig. 6). In our analysis, the quasi-diffuse transmitter is positioned as illustrated in Fig. 7(b). It emits a total average power $P_{\mathrm{trs}, \mathrm{ODIF}}$, which is equally divided among $M$ beams. The value of $M$ depends on whether the transmitter is of Type I or II, and on the horizontal transmitter-receiver separation $d_{h}$. For a Type I transmitter, $M$ is chosen so that for any position of the receiver within a circle of radius $d_{h}$ centered at the transmitter, at least one spot lies within the receiver FOV. Likewise, for a Type II transmitter, two

$$
P_{C, \mathrm{DIF}, \mathrm{SE}}=\int_{\substack{(x, y, z) \in \\ R_{C}, \mathrm{SE}}} \frac{3 A T_{F}(\psi) T_{C, \mathrm{SE}}(\psi) \sigma_{C} P_{\mathrm{trs}, \mathrm{DIF}} Z_{\mathrm{MAX}}^{5}}{2 \pi^{2}\left[\left(Z_{\mathrm{MAX}}-Z_{T}\right)^{2}+\left(x-X_{R}\right)^{2}+\left(y-Y_{R}\right)^{2}\right]^{2}\left[\left(Z_{\mathrm{MAX}}-Z_{T}\right)^{2}+\left(x-X_{T}\right)^{2}+\left(y-Y_{T}\right)^{2}\right]^{5 / 2}} d s
$$

$$
\begin{aligned}
& P_{\text {Wall,DIF,SE }}=3 A \sigma_{\text {Wall }} P_{\text {trs,DIF }} \\
& \times \int_{\substack{(x, y, z) \in \\
R_{\text {Wall,SE }}}} \frac{T_{F}(\psi) T_{C, \mathrm{SE}}(\psi) \sqrt{\left(x-X_{R}\right)^{2}+\left(y-Y_{R}\right)^{2}}\left(z-Z_{R}\right) \sqrt{\left(x-X_{T}\right)^{2}+\left(y-Y_{T}\right)^{2}}\left(z-Z_{T}\right)^{2}}{2 \pi^{2}\left[\left(x-X_{R}\right)^{2}+\left(y-Y_{R}\right)^{2}+\left(z-Z_{R}\right)^{2}\right]^{2}\left[\left(x-X_{T}\right)^{2}+\left(y-Y_{T}\right)^{2}+\left(z-Z_{T}\right)^{2}\right]^{5 / 2}} d s
\end{aligned}
$$


spots are required to lie within the receiver FOV. At each value of $d_{h}$, we find that the value of $M$ required for a Type II transmitter is close to twice that for a Type I transmitter. In the case of the Type I transmitter, the contribution from all $M$ illuminated signal spots is considered. By contrast, in the case of the Type II transmitter, one spot lying in the receiver FOV is randomly discarded, in order to emulate signal blockage (shadowing).

Each signal spot can overlap with regions of the ceiling and walls, which are assumed to act as Lambertian reflectors. We denote the region of the room boundary surfaces illuminated by the $m$ th spot by $R_{\text {Surface,spot, } m \text {. The average signal power re- }}$ flected by the room boundary surfaces is given by (20), shown at the bottom of the page, where $\sigma_{\text {Surface }}(x, y, z)$ is the reflectivity of the room surfaces, and the sum is taken over values of $m$ corresponding to unobstructed spots. Here, $\psi$ is given by (15), and $\phi$ is equal to $\psi$ if the surface is the ceiling, and equal to $90^{\circ}-\psi$ if the surface is a wall.

With a single-element receiver, the average detected signal power calculated using (20), omitting the factor $f_{i}(\psi, \theta)$ and replacing $R_{C, \mathrm{IMG}, i}$ with $R_{C, \mathrm{SE}}$, the region on the room boundary surfaces lying in the receiver FOV.

We consider transmission at $30 \mathrm{Mb} / \mathrm{s}$ using OOK. As shown below, at this bit rate, in the room depicted in Fig. 7, multipath ISI causes SNR penalties less than $1 \mathrm{~dB}(0.5 \mathrm{~dB}$ optical power penalty) in the three types of links considered. For each value of transmitter-receiver separation $d_{h}$, we consider a large ensemble of transmitter positions $(x, y)$ and azimuthal orientations (for the quasi-diffuse transmitter), and thereby estimate the ccdf of the SNR. We then use the ccdf to determine the transmitter power required to achieve a BER not exceeding $10^{-9}$ with $95 \%$ probability.

Fig. 8(a) shows the transmit power required in $30-\mathrm{Mb} / \mathrm{s}$ non-LOS links at transmitter-receiver separations $d_{h}$ of 4 and $8 \mathrm{~m}$, as a function of the number of pixels in the detector array. Type I quasi-diffuse transmitters, which do not provide immunity against shadowing, are considered. In the absence of shadowing, replacing a DIF/SE link by a QDIF/SE link reduces the power requirement by 1.2 and $3.8 \mathrm{~dB}$ at $d_{h}=4$ and $8 \mathrm{~m}$, respectively. Replacing a DIF/SE link by a QDIF/IMG link employing a 7-pixel receiver and MRC reduces the power requirement by 5.9 and $8.5 \mathrm{~dB}$ at $d_{h}=4$ and $8 \mathrm{~m}$, respectively. For a 1141-pixel receiver, the corresponding reductions are 14.3 and $16.2 \mathrm{~dB}$, respectively. In QDIF/IMG links, links using MRC always require less transmit power than SB. The advantage of MRC increases with the number of pixels, and is as hich as $2.6 \mathrm{~dB}$ for an imaging receiver with 1141 pixels at $d_{h}=8 \mathrm{~m}$.

Fig. 8(b) shows the transmit power requirements in $30-\mathrm{Mb} / \mathrm{s}$ non-LOS links, as a function of the transmitter-receiver horizontal separation $d_{h}$. Link designs include DIF/SE, QDIF/SE, and QDIF/IMG. QDIF transmitters are of Type I or Type II de-

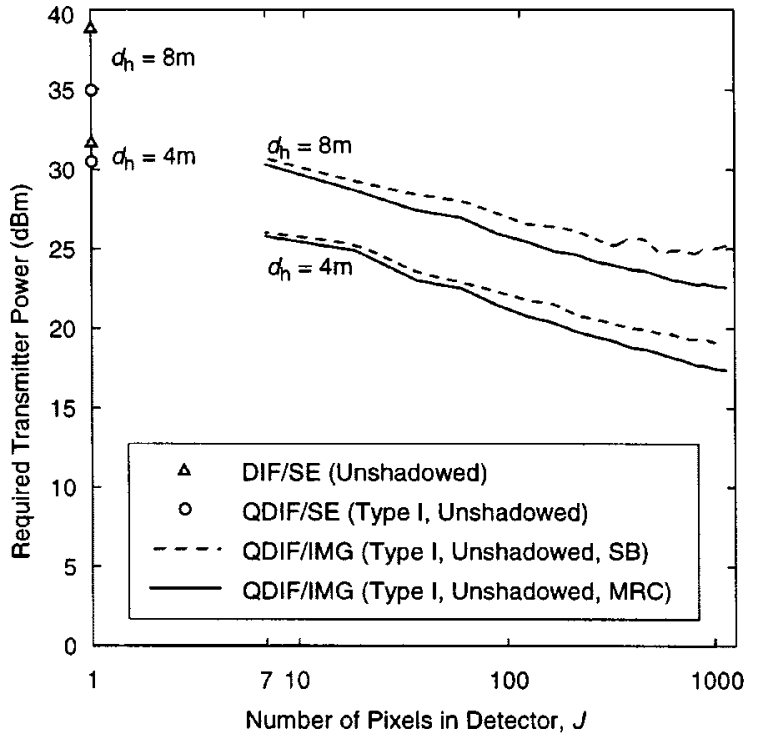

(a)

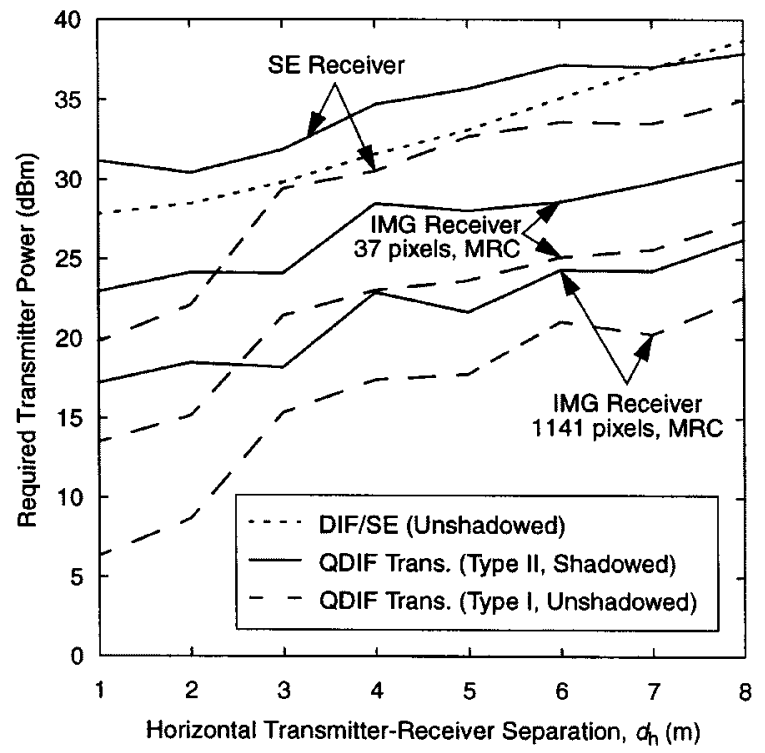

(b)

Fig. 8. Average transmitter power required to achieve a BER not exceeding $10^{-9}$ with $95 \%$ probability for DIF/SE, QDIF/SE and QDIF/IMG links. All links use on-off keying and operate at $30 \mathrm{Mb} / \mathrm{s}$. (a) With no shadowing, for various numbers of pixels in the detector array. Quasi-diffuse transmitters are of Type I design. Imaging receivers employ SB or MRC. (b) With and without shadowing, for various horizontal separations between the transmitter and receiver. Imaging receivers employ maximal-ratio combining. Unshadowed QDIF transmitters are of Type I design. Shadowed QDIF transmitters are of Type II design; to simulate shadowing, one signal spot within the receiver FOV is randomly discarded. Results with the diffuse transmitter do not take account of shadowing, which is expected to raise the transmitter power requirement by $2-5 \mathrm{~dB}$ over the values the values shown [4].

sign; in the latter case, shadowing of one signal spot is considered. The imaging receivers employ either 37 or 1141 pixels

$$
P_{\text {Surface, } \mathrm{QDIF}, \mathrm{IMG}, i}=\frac{P_{\mathrm{trs}, \mathrm{QDIF}}}{M} \sum_{m} \int_{\substack{(x, y, z) \in \\ R_{\text {Surface, } \mathrm{ppot}, m} \cap R_{\text {Surface,IMG }, i}}} \frac{A \sigma_{\text {Surface }}(x, y, z) T_{F}(\psi) f_{i}(\psi, \theta) T_{C, \mathrm{IMG}}(\psi)}{\pi\left[\left(x-X_{R}\right)^{2}+\left(y-Y_{R}\right)^{2}+\left(z-Z_{R}\right)^{2}\right]} \cos (\phi) \cos (\psi) d s
$$


with MRC. We first discuss results in the absence of shadowing. In this case, QDIF transmitters are of Type I design. Replacing a DIF/SE link by a QDIF/SE link reduces the transmit power requirement by $0.5-8.1 \mathrm{~dB}$, for $d_{h}$ between $1-8 \mathrm{~m}$. Replacing a DIF/SE link by a QDIF/IMG link with a 37-pixel receiver reduces the transmit power requirement by $8.5-14.4 \mathrm{~dB}$, for $d_{h}$ between 1 and $8 \mathrm{~m}$. For a 1141-pixel receiver, the power reductions range from 14.1 to $21.6 \mathrm{~dB}$.

In the presence of shadowing, we consider Type II quasidiffuse transmitters and block a randomly chosen signal spot within the receiver FOV. Examining Fig. 8(b), we note that for very small $d_{h}$, this leads to a large increases in the transmit power requirements, as compared to unshadowed links. The increases for QDIF/SE, 37-pixel QDIF/IMG, and 1141-pixel QDIF/IMG links are as large as $11.3,9.5$, and $10.8 \mathrm{~dB}$, respectively. Fortunately, larger values of $d_{h}$ are of practical interest. For values of $d_{h}$ of $3 \mathrm{~m}$ or larger, the increases in power requirement caused by shadowing for these three link designs are no more than 4.4, 5.4, and $5.5 \mathrm{~dB}$, respectively. Note that we have not calculated power requirements of DIF/SE links in the presence of shadowing. According to [4], shadowing typically increases the power requirements of DIF/SE links by $2-5 \mathrm{~dB}$. We conclude that for values of $d_{h}$ of interest, even in the presence of shadowing, QDIF transmitters and imaging receivers offer significant transmit power reductions.

3) Multipath-Induced ISI in Non-LOS Links: In our analysis of non-LOS links, we assume that the receiver does not equalize the received signal to mitigate the effects of multipath ISI. In this section, we quantify the impact of this ISI, following the derivations given in [20] and [4].

We assume the use of OOK at bit rate $B=1 / T$. In the $k$ th symbol interval, the transmitted symbol $a_{k} \in\{0,1\}$ modulates the amplitude of the transmitted pulse shape $A b(t)$, where $b(t)$ is a unit-amplitude rectangle of duration $T$. The transmitted signal passes through the channel with impulse response $h(t)$, and is passed through the receiver filter $g(t)$. The receiver filter $g(t)$ is chosen such that in the absence of multipath distortion, the received pulse will have a raised-cosine spectrum with $100 \%$ excess bandwidth. Decisions are made by the receiver based on the samples $y_{k}=A a_{k} \otimes h_{k}+n_{k}$, where $h_{k}$ is the link impulse response given by $h_{k}=\left.b(t) \otimes h(t) \otimes g(t)\right|_{t=k T}$ (assumed to have the normalization $\sum_{k} h_{k}=1$ ), and $n_{k}=\left.n(t) \otimes g(t)\right|_{t=k T}$ are zero-mean Gaussian noise samples with variance $\sigma^{2}$. The receiver sampling time is assumed to be shifted such that the zero-sample $h_{0}$ is maximized. As shown in [4], at low BERs, the optical power penalty caused by ISI is well approximated by

Optical Power Penalty (dB)

$$
\approx 10 \log _{10}\left(\frac{Q^{-1}\left(2^{L} \mathrm{BER}_{0}\right)}{\left(2 h_{0}-1\right) Q^{-1}\left(\mathrm{BER}_{0}\right)}\right)
$$

where $L$ is the length of the impulse response tail $\left(1-\delta_{k}\right) h_{k}$, $\mathrm{BER}_{0}$ is the desired BER, in our case $10^{-9}$, and $Q^{-1}(x)$ is the inverse of the function $Q(x)$ discussed previously.

We have used the numerical technique described in [20] to simulate the impulse response $h(t)$ of non-LOS links in the room shown in Fig. 7(a). Our simulations include the contributions from light diffusely reflected up to three times from sur-

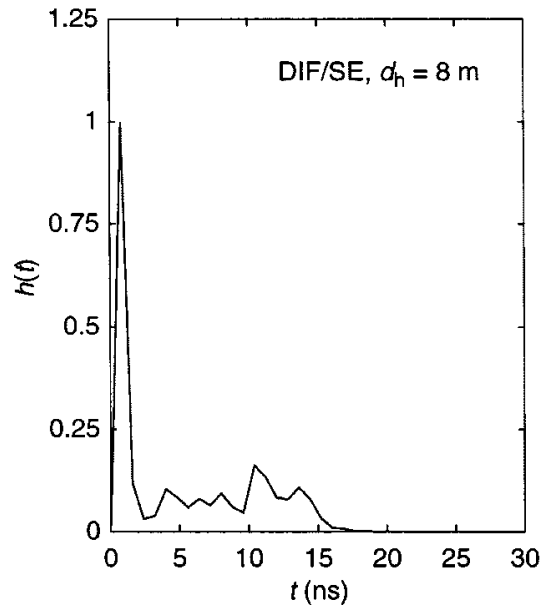

(a)

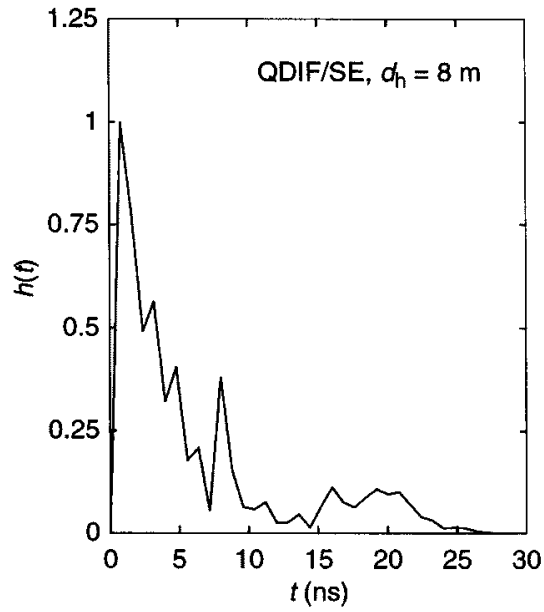

(b)

Fig. 9. Simulated channel impulse responses for the room used in our analysis. The single-element receiver is located at $(1.5,1.25,1)$, while the transmitter is located $8 \mathrm{~m}$ away and $1 \mathrm{~m}$ above the floor. Maximum values of the impulse responses have been normalized to unity. (a) Diffuse transmitter with $45^{\circ}$ half-power semiangle. (b) Quasi-diffuse transmitter with 18 beams.

faces within the room. Fig. 9(a) and (b) presents the impulse responses of DIF/SE and QDIF/SE for a transmitter-receiver horizontal separation $d_{h}=8 \mathrm{~m}$. Using (21) with impulse responses obtained within this room for $d_{h}$ up to $8 \mathrm{~m}$, we have found that for $\mathrm{BER}_{0}=10^{-9}$ and an optical power penalty due to ISI of $0.5 \mathrm{~dB}$ (1-dB loss in electrical SNR), the maximum allowable bit rate is $30 \mathrm{Mb} / \mathrm{s}$.

The channel impulse responses shown in Fig. 9 have been calculated for a single-element receiver. Use of an imaging receiver is expected to reduce the power penalty due to ISI considerably. This is because the delayed signals that cause ISI are received from a wide range of directions. Roughly speaking, these delayed signals are divided among a number of pixels that is of the same order as $J$, the total number of pixels. If the imaging receiver processes signals from three pixels, then the delayed signal amplitude should be of the order of $3 / J$ times that in a single-element receiver. Therefore, we can safely assume that with imaging receivers in the link configurations considered, the optical power penalty due to multipath ISI is below $0.5 \mathrm{~dB}$ at a bit rate of $30 \mathrm{Mb} / \mathrm{s}$. 


\section{SDMA}

In the context of IR wireless communications, optical multiplexing techniques allow simultaneous transmissions at the same subcarrier frequency and within the same space [3]. One form of optical multiplexing is SDMA, which involves the use of angle-diversity receivers to distinguish between signals received from different directions. SDMA can be implemented with imaging receivers and quasi-diffuse transmitters. Multiple quasi-diffuse transmitters form lattices of signal spots on reflective surfaces, such as a ceiling. Signal spots lying within the FOV of a receiver are imaged onto the receiver pixel array. It is hoped that the signal spots from different transmitters will image to disjoint sets of pixels (or nearly so), thus allowing the receiver to detect signals from different transmitters with acceptably small cochannel interference.

Below, we provide a simple analysis of the error probability of SDMA with two users, and describe a representative numerical example of a two-user system.

\section{A. Error-Probability Analysis for Two Users}

We assume that two receptions are incident within the receiver FOV. The desired and interfering receptions are designated by the subscripts 1 and 2, respectively. To simplify the analysis, the symbol clocks of the desired and interfering signals are assumed to be synchronized at the receiver, which represents the worst case. We assume that in both transmissions, the amplitudes 0 and 1 are equiprobable. The $i$ th pixel in the imaging receiver detects these two signals with average powers $P_{1, i}$ and $P_{2, i}$, respectively. This pixel has an additive Gaussian noise of variance $\sigma_{\text {tot }, \mathrm{i}}^{2}$, which is statistically independent of the desired and interfering signals. The combined noise and interference has a variance $\sigma_{\text {tot }, i}^{2}+r^{2} P_{2, i}^{2}$. The receiver implements MRC, wherein the signals from the $J$ pixels are combined using weights $w_{i}=r P_{1, i} /\left(\sigma_{\text {tot }, i}^{2}+r^{2} P_{2, i}^{2}\right), 1 \leq i \leq J$. We define the SNR for the desired signal as

$$
\mathrm{SNR}=\frac{\left(\sum_{i=1}^{J} w_{i} r P_{1, i}\right)^{2}}{\sum_{i=1}^{J} w_{i} \sigma_{\mathrm{tot}, i}^{2}}
$$

and the signal-to-interference ratio (SIR) as

$$
\mathrm{SIR}=\frac{\left(\sum_{i=1}^{J} w_{i} r P_{1, i}\right)^{2}}{\left(\sum_{i=1}^{J} w_{i} r P_{2, i}\right)^{2}} .
$$

The BER for reception of the desired signal is given by

$$
\begin{aligned}
\mathrm{BER}=\frac{1}{2} Q(\sqrt{\mathrm{SNR}}+ & \left.\sqrt{\frac{\mathrm{SNR}}{\mathrm{SIR}}}\right) \\
& +\frac{1}{2} Q\left(\sqrt{\mathrm{SNR}}-\sqrt{\frac{\mathrm{SNR}}{\mathrm{SIR}}}\right) .
\end{aligned}
$$

\section{B. Two-User Example}

We consider a numerical example involving two quasi-diffuse transmitters and an imaging receiver. Within the room shown in Fig. 7(a), the receiver and both transmitters face upwards, and are kept at a fixed height of $1 \mathrm{~m}$ above the floor. The $x$ and $y$ coordinates of the transmitters are modeled as two i.i.d. random

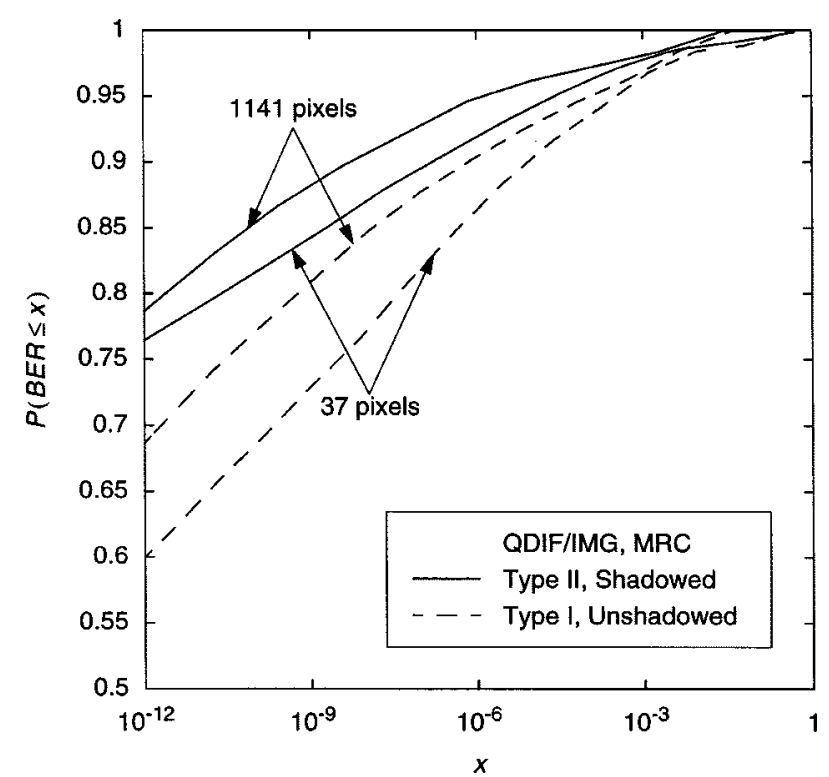

Fig. 10. Cumulative distribution function of the BER in an SDMA system with two quasi-diffuse transmitters and an imaging receiver. The position of each transmitter is uniformly distributed within a circle of radius $5 \mathrm{~m}$ centered at the receiver. Each transmitter emits an average power which, in the absence of cochannel interference, is sufficient to achieve a BER not exceeding $10^{-9}$ with $95 \%$ probability at a transmitter-receiver separation equal to $5 \mathrm{~m}$, as illustrated in Fig. 8(b). In the case of Type II transmitters, to simulate shadowing, one of the desired signal spots within the receiver FOV is randomly discarded. The receiver employs maximal-ratio combining.

variables, which are uniformly distributed within that portion of the circular region of radius $5 \mathrm{~m}$, centered at the receiver, that lies within the room. The imaging receiver is taken to have either 37 or 1141 pixels. We consider Type I transmitters, which are unshadowed. We also consider Type II transmitters, in which case, we randomly discard one of the spots of the desired transmission that lies within the receiver FOV. Each transmitter has sufficient power to achieve a BER not exceeding $10^{-9}$ with $95 \%$ probability in the absence of cochannel interference at a horizontal transmitter-receiver separation of $5 \mathrm{~m}$. As presented in Fig. 8(b), for Type I transmitters used with imaging receivers with 37 and 1141 pixels, the required powers are 231 and 59.7 $\mathrm{mW}$, respectively. For Type II transmitters, the required powers are 638 and $146 \mathrm{~mW}$, respectively. The symbol clocks of the desired and interfering signals are assumed to be synchronized at the receiver.

We have considered a large ensemble of different transmitter locations to estimate the cumulative distribution function (cdf) of the BER, which is shown in Fig. 10. It is seen that the performance is improved by using a Type II transmitter and/or a receiver having a larger number of pixels. A BER not exceeding $10^{-9}$ is achieved with a probability between $72.9 \%$ (unshadowed Type I transmitter and 37-pixel receiver) and $88.3 \%$ (shadowed Type II transmitter and 1141-pixel receiver). A BER not exceeding $10^{-6}$ can be achieved with a probability between $86.5 \%$ and $94.8 \%$ for these two link configurations, respectively.

Our results indicate that SDMA using quasi-diffuse transmitters imaging receivers may be a promising means to increase the capacity of IR networks. However, SDMA alone may not achieve reliability sufficient for many applications, particularly as the number of users increases. One way to enhance system 
reliability is to employ an adaptive, two-tiered multiplexing scheme. The default would be to employ only SDMA. When it is determined that the system is unable to achieve sufficient reliability using only SDMA, an additional, electrical multiplexing technique [3], such as time-division multiple access or subcarrier frequency-division multiple access, can be employed to achieve reliable operation. Future study of SDMA should address systems with multiple mobile transmitters and receivers and utilizing supplementary multiplexing techniques in conjunction with SDMA.

\section{CONCLUSIONS}

In this paper, we analyzed two modifications to the design of wireless IR links, which can dramatically improve link performance. Replacing single-element receivers by imaging receivers reduces received ambient noise dramatically and leads to the reduction of the required transmitter power by up to $13 \mathrm{~dB}$ in LOS links. In unshadowed, non-LOS links, replacing a diffuse transmitter by a quasi-diffuse transmitter can reduce the required transmitter power by over $8 \mathrm{~dB}$. Combining quasi-diffuse transmitters and imaging receivers in unshadowed, non-LOS links can reduce the required transmitter power by more than 20 $\mathrm{dB}$ compared to links using single-element receivers and diffuse transmitters.

We analyzed SDMA using quasi-diffuse transmitters and imaging receivers. We showed that with two transmitting nodes, a BER not exceeding $10^{-9}$ can be achieved with a probability close to $90 \%$ using SDMA. While further study of SDMA is warranted, these results indicate that SDMA using quasi-diffuse transmitters and imaging receivers is a promising means to increase the capacity of IR networks.

\section{REFERENCES}

[1] F. R. Gfeller and U. H. Bapst, "Wireless in-house data communication via diffuse infrared radiation," Proc. IEEE, vol. 67, pp. 1474-1486, Nov. 1979.

[2] J. R. Barry, Wireless Infrared Communications. Boston, MA: Kluwer, 1994.

[3] J. M. Kahn and J. R. Barry, "Wireless infrared communications," Proc. IEEE, vol. 85, pp. 265-298, Feb. 1997.

[4] J. M. Kahn, W. J. Krause, and J. B. Carruthers, "Experimental characterization of nondirected indoor infrared channels," IEEE Trans. Commun., vol. 43, pp. 1613-1623, Apr. 1995.

[5] I. Millar, M. Beale, B. J. Donoghue, K. W. Lindstrom, and S. Williams, "The IrDA standards for high-speed infrared communications," HewlettPackard J., Feb. 1998. Article 2.

[6] J. B. Carruthers and J. M. Kahn, "Angle diversity for nondirected wireless infrared communications," IEEE Trans. Commun., vol. 48, pp. 960-969, June 2000.

[7] R. T. Valadas and A. M. de Oliveira Duarte, "Sectored receivers for indoor wireless optical communication systems," in Proc. 5th IEEE Int. Symp. Personal, Indoor, and Mobile Radio Communications, The Hague, Netherlands, Sept. 21-23, 1994, pp. 1090-1095.

[8] C. R. A. T. Lomba, R. T. Valadas, and A. M. de Oiveira Duarte, "Sectored receivers to combat the multipath dispersion of the indoor optical channel," in Proc. Sixth IEEE Int. Symp. Personal, Indoor and Mobile Radio Commun., Toronto, ON, Canada, Sept. 27-29, 1995, pp. 321-325.

[9] A. M. R. Tavares, R. J. M. T. Valadas, and A. M. de Olveira Duarte, "Performance of an optical sectored receiver for indoor wireless communication systems in presence of artificial and natural noise sources," in Proc. SPIE Conf. Wireless Data Transmission, vol. 2601, Philadelphia, PA, Oct. 23-25, 1995, pp. 264-273.
[10] G. Yun and M. Kavehrad, "Spot diffusing and fly-eye receivers for indoor infrared wireless communications," in Proc. 1992 IEEE Conf. Selected Topics in Wireless Communications, Vancouver, BC, Canada, June 25-26, 1992, pp. 286-292.

[11] A. P. Tang, J. M. Kahn, and K. P. Ho, "Wireless infrared communication links using multi-beam transmitters and imaging receivers," in Proc. IEEE Int. Conf. Communications, Dallas, TX, June 23-27, 1996, pp. $180-186$.

[12] A. P. Tang, "Wireless infrared communication links using multi-beam transmitters and imaging receivers," Univ. California, Berkeley, M.S. Rep., Oct. 1995.

[13] J. M. Kahn, P. Djahani, A. G. Weisbin, K. T. Beh, A. P. Tang, and R. You, "Imaging diversity receivers for high-speed infrared wireless communications," IEEE Commun. Mag., vol. 36, pp. 88-94, Dec. 1998.

[14] D. Wisely and I. Neild, "A $100 \mathrm{Mbit} / \mathrm{s}$ tracked optical wireless telepoint," in Proc. 8th IEEE Symp. Personal, Indoor and Mobile Radio Communications, Helsinki, Finland, Sept. 1-4, 1997, pp. 964-968.

[15] A. Street, K. Samaras, D. O'Brien, and D. Edwards, "High speed wireless IR LAN's using spatial addressing," in Proc. 8th IEEE Symp. Personal, Indoor and Mobile Radio Communications, Helsinki, Finland, Sept. 1-4, 1997, pp. 969-973.

[16] J. R. Barry and J. M. Kahn, "Link design for nondirected wireless infrared communications," Appl. Opt., vol. 34, no. 19, pp. 3764-3776, July 1995.

[17] W. T. Welford and R. Winston, High Collection Nonimaging Optics. San Diego, CA: Academic, 1989.

[18] S. D. Personick, "Receiver design for digital fiber optic communications systems, I and II," Bell Syst. Tech. J., vol. 52, no. 6, pp. 843-886, July-Aug. 1973.

[19] R. G. Smith and S. D. Personick, "Receiver design for optical fiber communication systems," in Semiconductor Devices for Optical Communication. New York: Springer-Verlag, 1980.

[20] J. R. Barry, J. M. Kahn, W. J. Krause, E. A. Lee, and D. G. Messerschmitt, "Simulation of multipath impulse response for wireless optical channels," IEEE J. Select. Areas Commun., vol. 11, pp. 367-379, Apr. 1993.

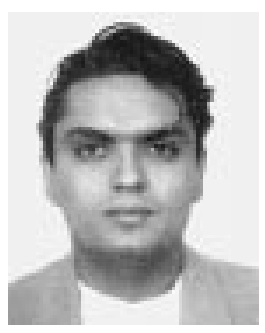

Pouyan Djahani received the B.A.Sc. degree in computer engineering from the University of Toronto, Toronto, ON, Canada, in 1994, and the M.S. degree in electrical engineering and computer sciences from the University of California, Berkeley, in 1998. His M.S. research involved the analysis of high-speed wireless infrared communications using multibeam transmitters and imaging receivers.

$\mathrm{He}$ is currently a Software Engineer, developing ATM communication software at Cisco Systems, San Jose, CA. From 1994 to 1996, he was a Development Analyst at IBM Microelectronics, developing software for infrared communication links, in particular IRDA links.

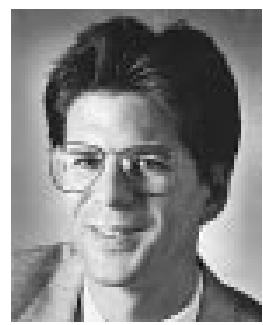

Joseph M. Kahn (M'87-SM'98-F'00) received the A.B., M.A., and Ph.D. degrees in physics from the University of California, Berkeley (UC Berkeley), in 1981, 1983, and 1986, respectively.

He is a Professor and Vice Chair for Graduate Matters in the Department of Electrical Engineering and Computer Sciences at UC Berkeley. From 1987 to 1990, he was Member of Technical Staff in the Lightwave Communications Research Department, AT\&T Bell Laboratories, where he performed research on multigigabit-per-second coherent optical fiber transmission systems and related technologies. He joined the faculty of UC Berkeley in 1990. His current research addresses several areas of communications, including wireless communication using antenna arrays, wireless communication for microelectromechanical systems (MEMS), optical wireless communication, and optical fiber communications.

Prof. Kahn received the National Science Foundation Presidential Young Investigator Award in 1991. He is a member of the IEEE Communications Society, the IEEE Information Theory Society, and the IEEE Lasers and Electro-Optics Society. He is serving currently as a Technical Editor of IEEE Personal Communications Magazine. 